\section{Long Sight}

I WAS at school at Rossall, between Fleetwood and Blackpool, on the coast of Lancashire. One day, being on the seawall with Arthur A. Dawson, an Irish boy, we could see the Isle of Man as if it were ten miles away, and then to the south of the Calf of Man we could distinctly see on the horizon the summits of two mountains, which we pronounced must be in Ireland. Four years later I was staying at Blackpool with my mother, when we distinctly saw the same blue mountains just appearing above the sea. Being in the Isle of Man later on, I was at Port Erie, to the west of Castletown, and saw the same summits, and was told they were the mountains of Mourne. From there the mountains stood well out of the water, though we could not see the rest of the coast. 'The Mourne Mountains are 2798 feet high. They are 125 miles from Blackpool.

Selsby Vicarage, Gloucestershire, May 28

A. Shaw Page

\section{Museums}

THE interest which the readers of NATURE in this country and in America take in the promotion of museums has induced so many of them to inquire of me for a paper recently noticed by yourself that, to spare their time and my own, I shall be glad if you will enable me to refer inquirers to your advertising columns.

The Author of "Museums of Natural Historv"

A NEW EXAMPLE OF THE USE OF THE INFINITE AND IMAGINARY IN THE SERVICE OF THE FINITE AND REAL

GEOMETERS are wont to speak (it seems to me) some$G$ what laxly of "the line at infinity" as if there were only one such line in a plane; in a certain but not in the most obvious sense this is true-viz. there is but one right line of which all the points are at an infinite distance from all lines external to them in the finite region of the plane, and except these points there are none others having this property; but in the sense that there is but one line infinitely distant from all points external to it in the finite region, the statement is obviously erroneous, for it need only to be mentioned to be at once perceived to be true by any tyro in geometry that all rays passing through either of the two "circular points at infinity" (Cayley's absolute) are infinitely distant from any external point in the finite region; these two imaginary points may indeed without any reference to the circle be defined as the points which radiate out in all directions rays infinitely distant from the finite region; the "absolute" being, so to say, the common depository, i.e. the crossing points of all infinitely distant rays as the "line at infinity" is the locus of all infinitely distant points. Similarly in space : there is not one infinitely distant plane, "the plane at infinity," but an infinitely infinite number of such planes-viz. any plane touching "the circle at infinity" (an imaginary circle in the plane at infinity) will at orce be recognised to be infinitely distant from any external point in the finite region, or, as we may say more briefly and picturesquely, infinitely distant from the finite region itself. It will give greater vivacity to this conception to imagine an axis through which pass planes in all directions, and to travel in idea this axis round "the circle at infinity" keeping it always tangential thereto ; the complex or corolla of planes, so to say, thus formed (infinitely infinite in number) contains only planes of infinite distance from the finite region; and "the plane at infinity" is but one of them-viz. the one which passes through all the axes named, just as the line at infinity in a plane is the line which passes through both the centres of infinite distance. The infinitely infinite series of infinitely distant planes is of course the correlative of the infinitely infinite series of infinitely distant, points whose locus is the so-called "plane at infinity."

The above statements have only to be made, to be accepted by the geometer, although I do not remember seeing them * anywhere explicitly given; but what I want to show is that, although supersensuous abstractions, so far from being barren they are capable of immediate application to the world of reality, and afford an instantaneous answer to a very simple practical question which has only just lately been mooted. The question is this: Suppose $a b c d$ to be a given pyramid, and that perpendiculars are drawn from its four vertices, say $A, B, C, D$, to a variable plane, then it is easy to show that a certain homogeneous quadratic function of $A, B, C, D$ C $\therefore$ pending on the form of the pyramid or relative lengths of its edges must be constant, and the question arises, What is this constant quadratic function, this quadric in $A, B, C, D$, expressed in terms of the edges of the pyramid exclusively? + Just so if we take a triangle, $a b c$, in a plane there will be a constant quadratic homogeneous function of the distances of its three vertices from a variable line; and it is well known in this case that if $A, B, C$ are the distances the constant quadratic function in question will be-

$$
\begin{gathered}
(a b)^{2}(A-C)(B-C)+(b c)^{2}(B-A)(C-A)+ \\
(c a)^{2}(C-B)(A B) .
\end{gathered}
$$

But if we had not known this fact it could have been found as follows:-Calling the above function $F$, when $A, B, C$ all or two of them become infinite the relation between the ratios of $A: B: C$ will be such as would arise from making $F=0$; on no other supposition will this be the case.

Now, if we use trilinear co-ordinates with $a b c$ as the triangle of reference, and take as the co-ordinates of any variable point $P$, the areas $a P b, b P c, c P a$ instead of the simple distances of $P$ from $a b, b c, c a$, then every body knows that the line at infinity has for its equation-

$$
\text { (1) } x+y+z=0 \text {, }
$$

and will easily see that the circle circumscribing $a b c$ has for its equation--

$$
\text { (2) }(a b)^{2} x y+(b c)^{2}(y z)+(c a)^{2} x z=0 .
$$

Moreover, when such co-ordinates are employed the distances of any line $A x+B y+C z=0$ (3) from the three vertices are $A, B, C$ each multiplied by the same known quantity.

If then $A, B, C$ become infinite this line must pass through one of the intersections of the line at infinity with the circle, or, in other words, the equations (1), (3), (2) must be capable of being satisfied simultaneously, and accordingly by a well-known algebraical law it follows that the determinant to (2) bordered by the coefficients of (I) and (3) must vanish. Consequently this determinant so bordered will represent the sought-for form $F$, i.e. the constant quadratic function will be represented by-

$$
\begin{array}{ccccc}
- & A & B & C & \bullet \\
A & \bullet & (a b)^{2} & (a c)^{2} & \mathbf{I} \\
B & (b a)^{2} & \bullet & (b c)^{2} & \mathbf{I} \\
C & \left(c a^{2}\right) & (c b)^{2} & \bullet & \mathbf{I} \\
- & \mathrm{I} & \mathbf{I} & \mathrm{I} &
\end{array}
$$

On calculating this determinant it will be found to be the function of $A-B, B-C, C-A$ above given, except that each term is multiplied by the constant factor -2 , which may of course be dispensed with.

Now let us apply similar or analogous considerations to the determination of the constant quadratic function of

* The statement concerning the circular point-pair at infinity being centres of pencils of infinitely distant rays I have since met with somewhere in Dr: Salmon's Conics, but stated in quite a casual manner. It may not be un worthy of notice that just as the distance between any two points in a ray passing through either point of the absolute in a plane vanishes, so similarly vanishes the area of any triangle drawn in any plane touching "the imaginary circle at infinity" in space.

$t$ If $l, m, n$. $p$ are the distances of the vertices from the opposite faces, and $x, y, z, t$ from the variable plane, it is well known that

$$
\Sigma\left(\frac{x^{2}}{l^{2}}-2 \frac{y z}{m n} \cos m, n\right)
$$

is constant, in fact is unity. 
$A, B, C, D$, the four distances on a variable plane from the fixed points $a, b, c, d$. I must promise that using quadriplanar co-ordinates $x, y, z, t$ analogous to those employed just now for the plane-viz. such as will cause

$$
x+y+z+t=0 \text { (1) }
$$

to become the equation to "the plane at infinity," the sphere circumscribing the fundamental pyramid $a b c a$ takes the analogous form to that given for the circle from which indeed it may be deduced with a stroke of the pen -viz. the equation to this sphere will be-

$$
\begin{gathered}
(a b)^{2} x y+(a c)^{2} x z+(a d)^{2} x t+(b c)^{2} y z+(b d)^{2} y t \\
+(c d)^{2} z t=0 \quad(2) .
\end{gathered}
$$

Moreover the distances of a plane whose equation is-

$$
A x+B y+C z+D t=0 \quad \text { (3) }
$$

from the vertices of the pyramid will be $A, B, C, D$ each multiplied by the same known quantity.

The intersection of the plane at infinity with any sphere, and consequently with the circumscribing sphere named, is "the circle at infinity;" hence if $F$ is the constant function required we may find it as the function of $A, B, C, D$, which becomes zero when the plane (3) is tangential to the intersection of the plane (I) with the sphere (2), or, which is the same thing, when the intersection of the planes (I) and (3) is tangential to the sphere (2), and this function is well known to algebraists to be the determinant formed by bordering the determinant to (2) with the coefficients of (1) and (3), i.e. we may take as the constant function $F$ the determinant following :-

$$
\begin{array}{cccccc}
\bullet & A & B & C & D & \bullet \\
A & \bullet & (a b)^{2} & (a c)^{2} & (a d)^{2} & \mathrm{I} \\
B & (b a)^{2} & \bullet & (b c)^{2} & (b d)^{2} & \mathrm{I} \\
C & (c a)^{2} & (c b)^{2} & \bullet & (c d)^{2} & \mathrm{I} \\
D & (d a)^{-} & (d b)^{2} & (d c)^{2} & & \mathrm{I} \\
\bullet & \mathrm{I} & \mathrm{I} & \mathrm{I} & \mathrm{I} & \bullet
\end{array}
$$

of which the developed value is easily found to be-

$$
\begin{gathered}
-\Sigma(a b)^{4}(C-D)^{2}+2 \Sigma(a b)^{2}(a c)^{2}(B-D)(C-D) \\
+2 \Sigma(a b)^{2}(c d)^{2}\left\{\begin{array}{c}
(A-C)(B-D \\
+(A-D)(B-C)
\end{array}\right\}
\end{gathered}
$$

This value of the constant function in its expanded form I some time ago found by a different method, and sent in the shape of a question to the Educational Times.* In a brief correspondence which ensued with Prof. Cayley, he wrote to me giving the equivalent determinant form which he arrived at by a totally different order of conceptions and in a very beautiful manner, as follows. We may regard the differences between $A, B, C, D$ as equal to the differences between the distances of $a, b, c, d$ from a fifth point, $e$, at an infinite distance, and may call $a e, b e, c e, d e$ equal to $A+K, B+K, C+K, D+K$ respectively, where $K$ is infinite. Hence by his own well-known theorem regarding mutual distances of five points we shall have--

$\left|\begin{array}{cccccc}\bullet & (a b)^{2} & (a c)^{2} & (a d)^{2} & (A+K)^{2} & \mathrm{I} \\ (b a)^{2} & \bullet & (b c)^{2} & (b a)^{2} & (B+K)^{2} & \mathrm{I} \\ (c a)^{2} & (c b)^{2} & \bullet & (c d)^{2} & (C+K)^{2} & \mathrm{I} \\ (d a)^{2} & (d b)^{2} & (d c)^{2} & \bullet & (D+K)^{2} & \mathrm{I} \\ (A+K)^{2} & (B+K)^{2} & (C+K)^{2} & (D+K)^{2} & \bullet & \mathrm{I} \\ \mathrm{I} & \mathrm{I} & \mathrm{I} & \mathrm{I} & \mathrm{I} & \bullet\end{array}\right|=0$

And by the ordinary well-known rules in determinants

* If the differences between $A, B, C, D$ be regarded as the minor determinants of the bilinear matrix $A \begin{array}{llll}A & C & D & \\ & & & \end{array}$ at once recognise that my form becomes expressible as a determinant of the 6th order, and I think I could hardly have failed eventually to have made this observation, the more especially as I was aware of the connection of the subject with that of the section of any sphere with the plane at infinity-but as a matter of fact Cayley anticipated me, and was the first to actually write down the function under the form of a determinant.

In each method the concept of infinity appears, but in mine that of the imaginary as well; and although more far-fetched than the other, the latter possesses the advantage of yielding the result as the transcript of a mere mental process without involving the necessity for the performance of any work whatever of algebraical reduction. for combining lines with lines and columns with columns it may easily be shown that the above determinant is of the form $4 F_{1} K^{2}+G K+H$, where $F_{1}$ represents-

$\begin{array}{cccccc}-\frac{1}{2} & A & B & C & D & \bullet \\ A & \bullet & (a b)^{2} & (a c)^{2} & (a d)^{2} & \mathrm{I} \\ B & (b a)^{2} & \bullet & (b c)^{2} & (b d)^{2} & \mathrm{I} \\ C & (c a)^{2} & (c b)^{2} & \bullet & (c d)^{2} & \mathrm{I} \\ D & (d a)^{2} & (d b)^{2} & (d c)^{2} & \bullet & \mathrm{I} \\ - & \mathrm{I} & \mathrm{I} & \mathrm{I} & \mathrm{I} & \bullet\end{array}$

Consequently $F_{1}=0$. This equation gives not only the form of the constant function but the value of the constant $\left(F_{1}\right.$, when the element $-\frac{1}{2}$ is suppressed, being identical with my $F$ ).

On removing the line and column of capital letters the above determinant equated to zero expresses the condition of the points $a, b, c, d$ lying in a plane--as proved by Cayley in days long past (and still ordinarily so proved) by a very artful manner of multiplying a determinant into a numerical multiple of itself; but this result follows as an instantaneous consequence of the reflexion that if $a, b, c, d$ did not lie in a plane the above equation would mean that the circumscriling sphere was touched by the plane at infinity, whereas we know that this plane never touches but has the faculty of always cutting every sphere in a constant circle of imaginary points. Hence the existence of this equation implies the coplanarity of the four points $a, b, c, d$, and the converse proposition may be shown by simple algebraical reasoning to follow from this.*

Postscript.-I have been led by what precedes to a rather interesting observation in universal geometry.

Suppose we form a determinant with the squared distances of one group of $n$ points from another equinumerous group any or all of which may be coincident with those of the former one: and to each line and at the foot of each column of this determinant affix a unit; a determinant so formed we may agree to call the bordered determinant of either group in regard to the other. Thus $e x . g r$.

$\begin{array}{cccc}a \boldsymbol{a}^{2} & a \boldsymbol{\beta}^{2} & a \gamma^{2} & \mathrm{I} \\ b \boldsymbol{a}^{2} & b \beta^{2} & b \gamma^{2} & \mathrm{I} \\ c a^{2} & c \beta^{2} & c \gamma^{2} & \mathrm{I} \\ \mathrm{I} & \mathbf{I} & \mathbf{I} & \end{array}$

is the bordered determinant of $a, b, c$ in regard to $a, \beta \gamma$. When the two groups are one group repeated we may call this determinant the bordered self-determinant of the groups.

My theorem is that the bordered determinant of two equi-numerous groups in respect to one another is a mean proportional to the bordered self-determinant of one of the groups, and that of the projection upon its niveau of the other group. [The nizeau to a group of points means the homaloid (Clifford's flat) of the lowest number of dimensions which contains the group.]

We may regard a group of $n$ points as the vertices of a figure whose squared content we know by Cayley's theorem above referred to is a sub-multiple of the bordered self-determinant of the group ; it is in fact that quantity divided by $(-)^{n+\mathrm{r}} 2^{n}(\mathrm{r} \cdot 2 \cdot 3 \ldots n)^{2}$, so that we may vary the statement of the theorem and say that the product of the contents of the figures denoted by two equi-numerous groups into the cosine of the inclination of their niveaus is a known numerical sub-multiple of the bordered determinant of one group in respect to the other. Thus, keeping at first within the limits of conceivable space, we see

* The equation in the text extended to the point $\mathrm{s} A, B, \ldots L, X$ assumes * The equation in the text extended to the point $\mathrm{s} A, B, \ldots$,
new importance and rises to philosophic interest when regarded as the intrinsic new importance and rises to philosophic interest when regarded asthe intrinsic equation to the nizeau of $A, B, \ldots L$, in which the co-ordinates of the
variable point $X$ in the niveau are the squares of $A X, B X, \ldots L X$; it is of course an equation of the second degree in these co-ordinates. The distances of either of two points from the other are the same in quantity but differ in sign. Hence the square of either is the natural measure of the interval between the two points. 
that the cosine of the angle between $a b c, a b d$, the faces of a tetrahedron, will be the determinant-

$$
\begin{array}{cccc}
\bullet & a b^{2} & a d^{2} & \mathrm{I} \\
a b^{2} & \bullet & b d^{2} & \mathrm{I} \\
c a^{2} & c b^{2} & c d^{2} & \mathrm{I} \\
\mathrm{I} & \mathrm{I} & \mathrm{I} &
\end{array}
$$

divided by sixteen times the product of the faces $a b c$, $a b d$.

Or, again, if $a b, c d$ be any two non-intersecting edges of the tetrahedron, $\pm 2 a b . c d \cos (a b, c d)$ ought to be equal to-

$$
\left|\begin{array}{ccc}
a c^{2} & a d^{2} & \mathrm{I} \\
b c^{2} & b d^{2} & \mathrm{I} \\
\mathrm{I} & \mathrm{I} & 0
\end{array}\right|
$$

and as a matter of fact the cosine between $(a b, c d)$ is equal to $\frac{a d^{2}+b c^{2}-a c^{2}-b d^{2}}{2 a b}$.

Again, if $a b c$, def are any two triangles in space of 5,4 , or 3 dimensions the product of their areas into the cosine of their inclination will be a numerical multiple of the bordered determinant of the group $a b c$ in regard to $d e f$, and if they lie in the same plane their product itself will be that numerical multiple.

Similarly for two groups of four points lying in one space (as ex gr. that in which we live, move, and have our being t) the product of their bordered self-determinants will be equal to the bordered determinant of either group in respect of the other, because their niveaus coincide, and if we take two groups of five points each in ordinary space it again follows from the theorem that the bordered determinant between them must vanish, a statement which when the two groups coincide reverts to Cayley's condition concerning the mutual squared distances of five points in ordinary space.

Finally, there can be little doubt, I think, of the truth of the following theorem dealing with determinants (but unbordered) $\neq$ of which the general theorem we have been considering which deals with bordered determinants must needs be a corollary.

By $P: Q$ where $P, Q$ are two groups of $n$ points each, let us understand the determinant formed by taking the cosines of the angles which the $n^{2}$ lines connecting $P$ and $Q$ subtend at a point $O$ equidistant, in space of the necessary number of dimensions, from each of the $2 n$ given points, and let $P^{\prime}, Q^{\prime}$ mean the groups $P$ and $Q$ augmented by the addition of $O$ to each of them, the theorem is that-

$$
\cos \left(P^{\prime}, Q^{\prime}\right)=\frac{P: Q}{\sqrt{(P: P)(Q: Q)}} \cdot \S
$$

* Obviously therefore we can express the squared shortest distance between two non-intersecting edges of a tetrahedron as a rational function of the squares of all six. The formula in the text is well known and easily proved fquares of all six. The formula in the text is well known and easily proved for the case of $a b c d$ heing in a plane, which is enough to show that it must
be true universally, for if we make $B C D$ rotate about $B C$, the projection be true universally, for if we make $B C D$ rotate about $B C$, the projection
of $C$ upon $B D$ does not move, and consequently $A C$ into the cosine of $A C$, of $C$ upon $B D$ do
$B D$ is invariable.

$B D$ is invariable.

† It would perhaps be more correct to say "which has its being in us."
t From which it follows that every algebraical theorem regarding square From which it follows that every algebraical theorem regarding square
matrices expressed in the umbral notation is immediately convertible into a matrices expressed in the umbral notation is immediately convertible into a proposition in universal geometry; the umbræ cease to be mere ab
and acquire a local habitation and a name as points in extension.

$\$ \sqrt{P: P}$ is in fact the factorial of $n$ divided by the $x$ th power of the distance of $O$ from each point in $P$ into the content of (what $I$ call) the plasm (of order $n$ ) denoted by $P$.

A plasm of the order $\mathrm{i}, 2,3$ means a rectilinear segment, a triangle, a tetrahedron-whence it is easy to deduce and define in exact terms the meaning of a plasm of any order as a figure bounded by plasms of the order next below its own. The squared content of a triangle is equal to the sun: of the 3 squared contents of its projections on mutually perpendicular planes in ordinary space : but also to the sum of the 6 squared contents of its projecthe square of the content of a plas $m$ denoted by $n$ points is similarly resoluble into a sum of $\frac{n(n-1) \cdots(n-i+r)}{1}$, such squares in extension of $(n+i-1)$ dimensions; as these squared contents are all expressible immediately by Cayley's theorem in terms of squared distances, the above statement gives rise to a far from self-evident theorem in determinants. What I
Thus for the case of $n$ equal to 2 if $O$ is the centre of the sphere passing through $a, b, c, d$, we ought to find the cosine of the angle between the arcs $a b, c d$ equal to

$$
\left|\begin{array}{ll}
\cos a c & \cos c d \\
\cos b c & \cos b d
\end{array}\right|
$$

divided by a square root of

$$
\left|\begin{array}{ll}
\cos a a & \cos a b \\
\cos b a & \cos b b
\end{array}\right|
$$

into a square root of

$$
\left|\begin{array}{ll}
\cos c c & \cos c d \\
\cos d c & \cos d d
\end{array}\right|
$$

i.e. equal to

$$
\pm \frac{\cos a c \cdot \cos b d-\cos a d \cos b c}{\sin a b \cdot \sin c d}
$$

as is the case.

There ought also to exist analogous theorems applicable to non-equi-numerous point groups depending in some way upon the minors of a corresponding rectangular matrix.*

$$
\text { New College, Oxford, April } 1885
$$

J. J. SYLVESTER

\section{GRESHAM COLLEGE}

THE question of what is to be done with one of the greatest of existing London abuses, Gresham College, has again come up in connection with a letter from a "Londoner" in the Times. The Times, in a somewhat incomplete leader, animadverts strongly on the abuse, and urges its prompt remedying. Surely when the fact that London has no university in the true sense is attracting so much attention and the movement to supply the want is so powerful, it is absurd to allow the funds to be worse than wasted which represent the wreck of those which were originally intended for the maintenance of a real institution of this class. There were once 20,000 students at Gresham College, and when London does have a university, as it must have some time, even Gresham College will be without raison d'être.

"Topographically," the Times says, "the lecture-rooms are off the track of students. None of the apparatus of systematic instruction, in the way of examinations, accompanies the courses. Provision does not exist, have here termed plasms might with more exactitude be termed protoplasmis, as being the elements into which all other figures are capable of being resolved.

* It may be objected that the theorems of the text applied in their full generality beyond the limits of empirical space cease to affirm a relation between two different things and therefore lose their efficacy as such and become mere definitions of the meaning of the inclination of two figures in supersensible space. To meet this objection it is sufficient to give a general method for determining algebraically the projection of a point in space of $n$ dimensions on the niveau of ${ }^{\prime}$ points where $v$ is any number not greater than $n$; this it is easy to see may be effected as follows :-

(a) I observe that the niveau of any $\mu$ given points in a space of $n$ dimensions may be expressed in Cartesian co-ordinates by means of equating to zero each of $n-\mu+1$ independent minors of a rectangular matrix containing
$n+1$ columns and $\mu+x$ lines, the formation of which is too obvious to need $n+1$ columns and
stating in detail.

stating in detail.
$(\beta)$ In order to project orthogonally a point whose $n$ co-ordinates in a space of $n$ dimensions are $x^{\prime}, y^{\prime}, \ldots z^{\prime}$ upon a niveau (of the $(n-1)$ th order) passing through $n$ given points defined by the equation $A x+B y+\ldots C z+L=0$ we have only to write $x-x^{\prime}: y-y^{\prime}: \ldots: z-z^{\prime}:: A: B: \ldots: C$, and combining the $(n-I)$ equations contained in this proportion with the given equation, the resulting values of $x, y, \ldots z$ determine the projection of the given point on the given niveau.

If now $v$ points are given in a space of $n$ dimensions and the projection is required of a given point upon their niveau we may proceed as follows :-

(x) Find the $n-v+x$ equations which define the niveau.

(2) On each of the niveaus of the $(n-1)$ th order which correspond thereto respectively find the orthogonal projections of the given point.

(3) Through these $n-v+1$ projections of the given points and the given point itself draw a niveau which will be defined by $(n+1)-(n-\nu+2)$, i.e. $y$ - I equations.

Finally, combining these with the $n-v+\mathrm{r}$ original equations we have $n$ equations in all, and these will serve to determine the $n$ co-ordinates of the projection required.

projis method is not always the most compendious, but is always sufficient,
The and enables us to attach a definite meaning to the inclination of two spaces of and enables us to attach a definite meaning to the inclination of two spaces of any the same order to one another : thus ex. gr., the content of the pro-
jection of $a b c d$ on $e f g h$ divided by the content of $a b c d$ itself is the cosine of the inclination of the niveaus $a b c d, e f g h$, and the projections of cosine of the inclination of the niveaus $a b c d, e f g h$, and the projections of
the several points $a, b, c, d$ on $e f g h$ (say $\left.a^{\prime}, b^{\prime}, c^{\prime}, d^{\prime}\right)$ being found by the the several points $a, b, c, d$ on $e f g h$ (say $a^{\prime}, b^{\prime}, c^{\prime}, d^{\prime}$ ) being found by the
preceding method, the content of the tetrahedron $a^{\prime} b^{\prime} c^{\prime} d^{\prime}$ (and therefore preceding method, the content of the tetrahedron $a^{\prime}$
the inclination of the two niveaus) is a known quantity. 